\title{
Time-of-use Short-term Load Prediction Model Based on Variable Step Size Optimized HBMO-LS-SVM Method in Day-head Market
} Guo Lei ${ }^{a}$, Xue Song ${ }^{a^{*}}$, Liu Yang $^{b}$, Zeng Ming ${ }^{b}$

${ }^{a}$ State Grid Energy Research Institute, Changping District, Beijing 102209, China

b State Key Laboratory of Alternate Electrical Power System with Renewable Energy Sources, North China Electric Power University, Changping District, Beijing 102206, China

*Corresponding author: Xue Song, Doctor of Administration, xuesongbjhd@163.com

\begin{abstract}
The short-term load prediction results are important basis for arranging dispatching plans scientifically, decision-making for competitive bidding of power generation enterprises and users. Firstly, this paper constructs a factor index system of time-of-use short-term load prediction of next day in day-head market, and then builds the HBMO-LS-SVM prediction model. In order to prevent falling into local optimum traps, it optimizes the prediction model based on variable step size, which can improve the convergence speed of prediction as well as prediction accuracy in principle.
\end{abstract}

Key words: time-of-use short-term load prediction; variable step size optimization; HBMO-LS-SVM algorithm

\section{Introduction}

Time-of-use short-term load prediction results are the important basis of decision-making in power generation enterprises and power-purchasemain body making day-head analysis on next day supply-demand equilibrium situation besides power grid enterprises arranging dispatching plans. It has strong practical significance for improving the market main body decision-making ability to improve time-of-use short-term load prediction accuracy. Currently time-of-use short-term load prediction is mainly through artificial neural networks method without considering the impact of power price market factors on the power price ${ }^{1}$. At the same time, the artificial neural networks method exists discrete to some extent, which is easy to fall into local optimum traps.

This paper firstly constructs date property data samples, meteorological data samples, historical load data samples, historical power price data samples and other sample series; Secondly, in order to improve data simples' quality, it builds bad data detection and handling model respectively from vertical and horizontal dimensions. Finally, this paper constructs HBMO-LS-SVM short-term power price prediction model to overcome the shortcomings of traditional time-of-use short-term load prediction method and it optimizes the prediction model based on variable step size, which avoids falling into local optimum traps effectively and can improve the convergence speed of prediction as well as prediction accuracy.

\section{Time-of-use load factors simple data structure}

Arrange the load sequence according to different time points into 24 power price series(namely time-of-use power price sequence, if the market-clearing happens every half an hour, there are 48 power price series.)Factors which affect time-of-use load can be divided into date property, meteorological factor and historical load factor. Integrating the existing 
research results, this paper also includes historical power price factor, considering the incentives of power price to power generation main bodies and users. It constructs sample data structure comprising four categories factors, and the specific index system are shown in Fig. 1.There area lot of research of specific factors sequence expression which are shown as literature $^{2,3}$.

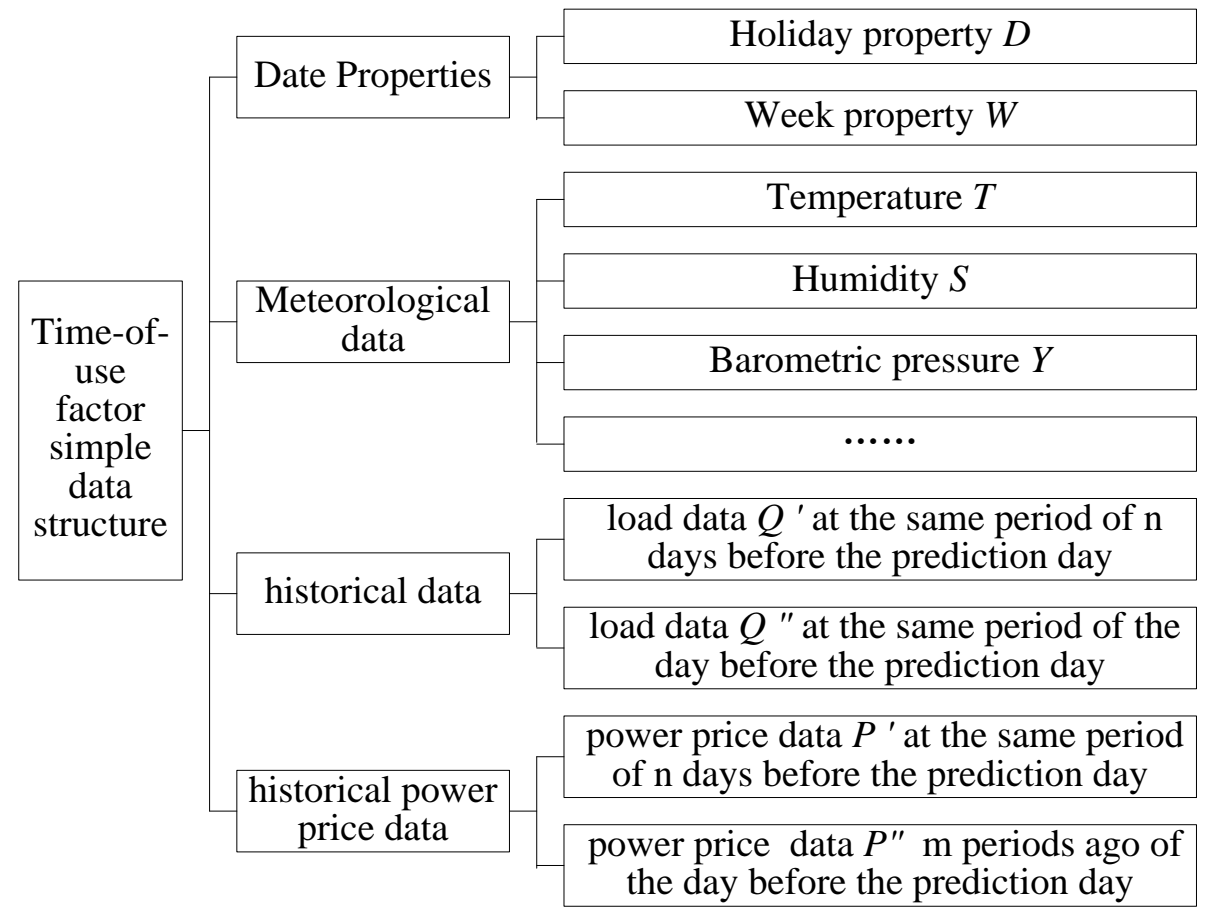

Fig.1- Time-of-use load factor simple data structure

Vertical and horizontal detection and handling method of bad data see reference ${ }^{4}$.

\section{(1) Vertical detection and handling of bad data}

There are similarities among sample data at the same period in different days, and the value should be maintained within a certain range. If the value exceeds this range, it is regarded as bad data. The formulas are shown as follows:

$$
|y(i, t)-\bar{y}(t)|>\varepsilon(t)
$$

Then

$$
y(i, t)=\left\{\begin{array}{l}
\bar{y}(t)+\varepsilon(t) y(i, t)>\bar{y}(t) \\
\bar{y}(t)-\varepsilon(t) y(i, t)<\bar{y}(t)
\end{array}\right.
$$

Wherein: $\bar{y}(t)$ is load average value at the time $t$ in recent days.

$y(i, t)$ is load value at time $t$ in $i$-th day.

$\varepsilon(t)$ is threshold, which can be set artificially.

\section{(2) Horizontal detection and handling of bad data}

Take the same sample data from two neighbor periods as a reference, and set the maximum data variation range. If both the value and the absolute value of the difference between the 
two neighbor periods exceed the threshold, it is identified as bad data. The formulas are shown as follows:

$$
\left\{\begin{array}{l}
|y(i, t)-y(i, t-1)|>\alpha(t) \\
|y(i, t)-y(i, t+1)|>\beta(t)
\end{array}\right.
$$

Then

$$
y(i, t)=\frac{y(i, t+1)+y(i, t-1)}{2}+y(i-1, t)-\frac{y(i-1, t+1)+y(i-1, t-1)}{2}
$$

Wherein, $\alpha(t), \beta(t)$ are the thresholds.

\section{Short-term power price prediction model based on variable step size optimized HBMO-LS-SVM optimization algorithm}

\subsection{LS-SVM model}

SVM can solve the practical problems of small sample, nonlinear, high dimension and local minima. It has been successfully applied to the classification, function approximation, and time series prediction and so on.Compared with the standard SVM model, the advantages of LS-SVM method are :equality constraints instead of inequality constraints of standard SVM algorithm; the solving quadratic programming problem converted to a direct solution of linear equations. The specific calculation model is shown in the literature ${ }^{5}$.Training LS-SVM can find kernel parameters as well as the regularization parameter,which has an important influence on the accuracy of LS-SVM model. These parameters by transforming stepscan be called directly in HBMO optimization training phase.

\subsection{HBMO Algorithm}

HBMO optimization algorithm is one of colony algorithms, belonging to the category of swarm intelligence. The main steps of bee mating optimization algorithm are:

Step one: The queen flies out of the nest after sexual maturity and selects a drone to form spermatheca probabilistically.

Step Two: The queen chooses a drone to foster larvae.

Step three: The worker bees complete local search heuristics.

Step four: The worker bees arrange to foster larvae according to its growth.

Step Five: After every mating, the flight speed of the queen appears kind of deduced, and when the queen is weakened to a certain extent, the mature and competent larvae replace to it. The whole process produces a new generation queen, who cross-links the gene of drone and the original queen, in that case the life cycle of original queen will end. Bees' breeding evolutionary process is the process of queen updating constantly. The generating of new queen is similar to the evolutionary computation in the optimization process, and the queen is the optimal solution of the problem to be solved in the optimization process.

According to the steps of bees algorithm, specific model finding the optimal kernel parameter and the regularization parameter is shown in literature ${ }^{6}$.

\subsection{Improved HBMO-LS-SVM Prediction Model based on variable steps}

Important characteristics where HBMO algorithm outperforms genetic algorithm are that each iteration of HBMO algorithm conducts local search until find the global optimal solution after 
a sufficient number of iterations. However, when the best individual is not changing generationally, the algorithm will fall into a local optimal trap. In order to solve the problem of easy falling into local optimum, it needs to introduce variable step size to the population in order to get rid of local optimum and to start a new search.

Improved HBMO-LS-SVM method based on variable step size is shown in Figure 2.

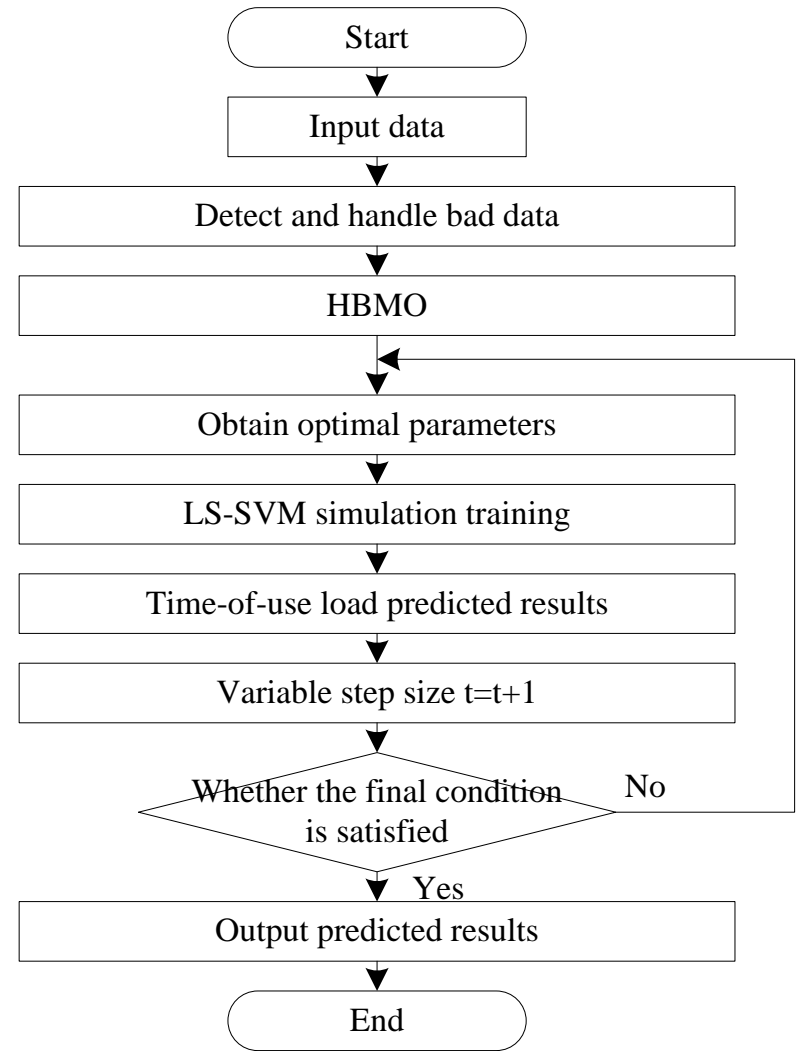

Fig.2. Algorithm flow of improved HBMO-LS-SVM short-term power price prediction model based on variable steps

\section{Conclusion}

With the new round reform of the power market as well as the establishment and improvement of the spot market, accurate time-of-use load prediction has a strong practical significance in China, and is able to provide support for both transaction sides of power acquisitionand sale to make scientific and rational bidding decision-making. The main innovations of this paper are increasing historical power price data in the time-of-use shortterm load prediction, conducting bad data detection and handling before training samples, doing prediction with variable step size optimized HBMO-LS-SVM algorithm. Theoretically it is possible to improve the prediction accuracy as well as convergence rate, and is expected to achieve a perfect prediction result.

\section{Acknowledgements}

The work described in this paper was supported by Science and technology projects of State Grid Corporation (No. SGERI06KJ [2015]63). 


\section{References}

1. L.Li, J.Wei, C.B.Li, et al. Prediction of Load Model Based on Artificial Neural Network, Transactions of China Electrotechnical Society, 8(2015) 225-230.

2. Y.P.Zhu, G.F.Fang, Short-term load forecasting based on dynamic adaptive artificial neural network and human body amenity indicator, Power System Protection and Control, 1(2012) 56-61.

3. X.Y.Wu, J.H.He, P.Zhang, et al. Power System Short-term Load Forecasting Based on Improved Random Forest with Grey Relation Projection, Automation of Electric Power Systems, 12(2015) 50-55.

4. Y.Cai, Research on Short-term Load Forecasting Based on Support Vector Machine[D]. Wuhan: Huazhong University of Science and Technology, 2007.

5. M.Zeng, Lv Chunquan, Tian Kuo, et al. Least Squares-support Vector Machine Load Forecasting Approach Optimized by Bacterial Colony Chemotaxis Method, Proceedings of the CSEE, 34(2011) 93-99.

6. M.Zeng, S. Xue, Z.J.Wang, et al. Short-term Load Forecasting of Smart Grid Systems by Combination of General Regression Neural Network and Least Squares-Support Vector Machine Algorithm Optimized by Harmony Search Algorithm Method, Applied Mathematics \& Information Sciences, 7-1L(2013) 291-298. 